\title{
Progress of computer potential of mankind as the basis for a new model of the universe
}

\author{
Pavel A. Stabnikov
}

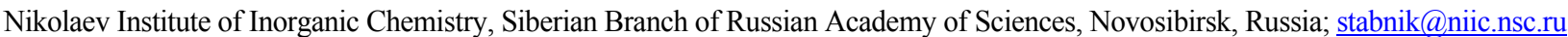

Received 13 August 2010; revised 20 September 2010; accepted 23 September 2010.

\section{ABSTRACT}

Known models of the Universe development are discussed in the present work. At present it is not possible to state what model is true among the suggested ones because all models are based on the assumptions a validity of which is scarcely determined. There are plenty of reasons, and the most important ones are impossibility of experiments performance on a global scale, very short time of nature study and low accuracy of determination of fundamental physical constants to check their possible drift. In the most of models the intellect is an unnecessary attribute. Mankind is only an inner insignificant observer in the Universe. Small changes which Humanity can produce on the Earth don't modify our planet on a global scale. However besides changes in the material world Mankind can create intellectual valuables. Large information content can be stored, integrate and process using computers. We have no any principle restrictions in advance of the computer engineering. Therefore we propose a new model of the Universe development which is based on the increasing facilities of our Mankind. In this model the Earth is considered as an analogue of supercomputer. Under certain circumstances Mankind is ready to carry out information processing for other civilizations, but such civilizations have not yet been found. The Creators of the Universe could be other customers. Computer-like model of the Universe suggests a communication with the Creators in future for execution of their calculation orders. However this model is not complete because of lack of high rate of information transfer for long distances.

Keywords: Universe Models; Matter and Information; Mankind Facilities; Computer Models

\section{INTRODUCTION}

To date, a huge number of models of the Universe development were proposed, which are based on the physical and cosmological data [1-5]. Because of the low accuracy of some physical constants, but also because of the impossibility of conducting experiments on a global scale, all such models are still only proposals. In most well-known hypothesis of the Universe the mind is unnecessary attribute. The main purpose of this paper is to describe the new, computer-like model of the Universe development, where we use the physical characteristics of the surrounding world, as well as increasing possibilities of the Mankind (cognition of nature, information processing, creation of models, theories and systematology). In developing the model, we tried to answer the pragmatic aspects of the nature: who created our world and what, eventually, humanity will be able to do in the future. We are attracted to the idea, borrowed from the religious, scientific, and popular fiction. These ideas are recycled in accordance with the modest capabilities of humanity to change the material world on a global scale and progress in information processing. These ideas are fastened by a pragmatic approach, inspired by the energy shortage that already constrains the further development of human civilization. Suggested computer-like model of the Universe in the future involves communication with the Creators to do their computing orders.

\section{COSMOLOGICAL MODELS OF THE UNIVERSE}

All the proposed hypothesis of the universe can be divided into two main groups:

1) The first group includes the hypotheses assuming that the matter will continue scattering forever. Broadening may be either uniform or accelerated $[1,2]$. The average density will decrease permanently. Galaxies, starts, planets, atoms will be gra- dually decomposing. Only the energy will remain in the infinite space. In these hypotheses, 
Mankind just plays a part of an interior indifferent observer for a small time interval. Moreover, mind is an unnecessary attribute of the Universe that in no way can affect the general motion of the matter.

2) The second group includes the hypotheses assuming that scattering and rapprochement of the matter occur along a sinusoidal curve or a helix $[3,4]$. In these hypotheses, the notion of the Big Bang is replaced by Big Bounce, or the matter in whole is represented as a kind of a spring that oscillates from the most dense to the most rarefied state. In these hypotheses, the role of Mankind is also just an observer. This group also includes the hypotheses in which the development of the Universe proceeds along a broken line as an infinite saw. These are the so-called pulsating models of the Universe. The start is the Big Bang, then scattering of the matter proceeds until a definite density is achieved; then a new bang occurs. Big Bang may be initiated either by a supernova or by an explosion organized by a civilization of humanoids. It is this latter case when the civilization may become a primer of a new Big Bang. If we adhere to this hypothesis, Mankind will sooner or later become the initiator of a new Big Bang. However, this may happen only if another civilization developing somewhere in Universe fails to leave us behind in this deed. However that may be, it has been established experimentally by present that the explosions of atomic bombs do not cause a new Big Bang. It remains unclear whether experiments at the Large Hadron Collider will be able to initiate a new Big Bang. We will get aware of this within the nearest 10 years (May be 12/21/2012. This date in the predictions of the ancient Mayan completes the era of the "Fifth Sun").

It should be noted that only few hypotheses put forward the idea of a steady unvarying Universe. The reason is that the soviet mathematician A. Fridman demonstrated in 1922-1924 with the help of the theory of A. Einstein that the development of the Universe may follow two routes: expansion or compaction. The Universe that is steady on the global scale would not be stable. In addition, some authors assume the existence of many universes, similar to our Universe, but with possibly different physical laws [2,5].

But why has it not been established yet what hypothesis among the proposed ones is the most correct? There are several reasons; the major ones are: impossibility to perform experiments on the global scale, short period of time (in the universal scale) during which the nature has been under investigation, low accuracy of determination of some physical values. For the present time, this does not allow us to establish the presence or the absence of interconnection between definite physical parameters during the motion of material objects for long time intervals. Thus, the velocities with which the galaxies move away are calculated according to Doppler Effect on the basis of the red shift value in the spectra of starts in those galaxies. However, in order to recalculate the shift values into the velocities of galaxies moving away, we are to assume that Doppler Effect on the intergalactic scale is also connected mainly with the velocity of bodies moving nearer or away, as it is on the Earth's scale. We cannot confirm this assumption with any experiments, so we cannot reject also other hypotheses, for example, that light passing through intergalactic distances becomes aged, so that its frequency decreases, or the velocity of the light increases permanently, due to which its frequency may be decreasing, while galaxies do not move away at all, or they do move but with smaller velocities. In addition, the light from remote galaxies was radiated by the atoms of starts several hundred million years ago, but we compare it with the spectra recorded today. Because of this, before making comparisons, we should additionally assume that the spectra remained unchanged during this time also on the Earth. The correctness of assumptions may become clear only in the distant future.

In addition, we do not have any data showing how stable the fundamental physical constants are during long intervals of time. According to definitions, these values should be invariable. However, some physicists doubt that these constant are invariable. For example, P. Dirac formulated a hypothesis in 1937 that the gravitation constant may decrease while the Universe develops. Consequences of the changes of some fundamental physical constants for the Universe are discussed in $[5,6]$. At present only physical constant known to be changed with time is Hubble constant. The point is that the age of the Universe $\mathrm{T}_{0}$ and the value of the Hubble constant $\mathrm{H}_{0}$ are connected with each other [6] through the equation:

$$
\mathrm{T}_{0}=1 / \mathrm{H}_{0} \quad \text { or } \quad \mathrm{T}_{0} * \mathrm{H}_{0}=1
$$

This is true if $\mathrm{T}_{0}$ and $\mathrm{H}_{0}$ are expressed in the same units, for example in seconds and reciprocal seconds, respectively. In the general case, this equation is written as $\mathrm{T}_{0} * \mathrm{H}_{0}=\mathrm{k}$, where $\mathrm{k}$ is a coefficient depending on the units in which $\mathrm{T}_{0}$ and $\mathrm{H}_{0}$ as expressed. According to the newest data, the age of the Universe is (13.72 \pm 0.12$)$ $10^{9}$ years, or $(13720000 \pm 120000)$ thousand years. After 1 thousand years, its age will increase by a unit in the eighth significant digit. Thus $\mathrm{H}_{0}$ should decrease by a corresponding value also in the eighth significant digit. Today we have $\mathrm{H}_{0}=(74.2 \pm 3.6) \mathrm{km} / \mathrm{s} / \mathrm{Mparsec}$, only 
two significant digits. If one day in the future it will be possible to determine $\mathrm{H}_{0}$ with the accuracy up to eight significant digits, 1000 years later it would be possible to establish the drift of Hubble constant in time. If people would become able to determine $\mathrm{H}_{0}$ with the accuracy up to nine significant digits, the same procedure will take only 100 years. But what will happen with the red shift values after 1000 years? On the one hand, $\mathrm{H}_{0}$ will decrease; on the other hand, the distances from the galaxies will increase during that time, so the red shift values will remain the same. This will be so only in the case if the model of Universe expanding uniformly (without acceleration) is true. In addition, the spectra of atoms, recorded on the Earth, should remain unchanged, too.

By present, CODATA (Committee on Data for Science and Technology) recommends (according to [7]) the values of Rydberg constant to 13 significant digits, proton mass to 11 significant digits, electron mass, electric constant, Planck constant to 10 significant digits, the velocity of light and some other fundamental physical constants to 9 significant digits. The majority of fundamental physical constants have been determined with the accuracy of 5, 6, 7 significant digits, so it is difficult at present to determine their possible drift. In order to establish which of the constants are indeed constant and which change, it is necessary to organize a supervisory service, but this service should operate more than a century.

So, due to the impossibility of performing additional experiments on a global scale, or due to the short time interval during which the fundamental physical constants and their accuracy are monitored, in many cases we cannot confirm or reject one or another model of the Universe. This is one of the paradoxes of modern cosmology. If we are unable to demonstrate experimentally the falseness of a hypothesis, we should consider it plausible. These are the general principles of science development, as the science develops due to any ideas and hypotheses including the craziest ones. Here we are to adhere to a simple idea: the larger is the number of hypotheses dealing with the development of the Universe, the higher is the probability that anyone of them will turn out to be true. Today we may only suppose that a model is more plausible in comparison with others.

Now we will venture to give an advice how to propose a model of the Universe which could not be easily denied: that model should rely on the new ideas and hypotheses, as well as on the data obtained during the recent years. The model should not contradict easily verifiable experimental data. As far as the behavior of galaxies or atoms at long distances and during long time intervals is concerned, assumptions may be fantastic. In any case, it would be impossible to confirm or reject them during the forthcoming thousand years. To decorate the model, we may introduce the drift of some fundamental physical constants depending on the astrophysical parameters of the model. For instance, it was assumed in [3], proposing a model of the cyclic development of the Universe, that the Universe would expand until the masses of electron and proton become equal to each other. The authors of [3] call this moment the degeneracy point. Then the galaxies wills tart to approach each other, and the masses of electron and proton will change in the reverse direction subsequent 15 milliard (billion) years. However, the mechanisms through which the mass gets redistributed between the proton and the electron is not discussed in that work. In all other respects, this is rather beautiful model that cannot be either confirmed or rejected at present.

In addition, any model proposed for the development of the Universe may be decorated with quite unusual assumptions and notions. We should mention [1] introducing the notion of Newtonian antiattraction for the assumed accelerated expansion of the Universe; the authors of [2] introduce the notion of the "dark energy" for the same purpose. If it turns out that, quite contrary, the expansion of the Universe slows down, then antigravitaiton may be replaced with hypergravitation; there is no need to change the term "dark energy" because nobody knows what it is. We should also mention the wormholes [8], the presence of which should allow Mankind to reach distant points, both in our world and in the possible parallel worlds.

Following these recommendations, we may propose a large number of the models of the Universe. Below we will propose a hypothesis of the development of the Universe that rests upon the astrophysical data and on the possibilities of Mankind in the reclamation of nature and in information processing. To start, we will discuss what the information is.

\section{MOTION OF THE MATTER AND INFORMATION}

The definition of information, contained in the philosophical [9] and the Polytechnic [10] dictionaries, from our point of view, are incomplete and one-sided. So here, based on these definitions, we will offer a more complete interpretation of the term information.

The most general categories in our world are matter, information, ideal. We proceed from the opinion that the matter is prime. It is usually stated that the matter is permanently moving and changing; this motion is independent of whether we or anybody else have any notion of this motion or no. But this motion is not absolute. Some material objects may remain unchanged during long time intervals, which give us the possibility to store 
information. The matter also possesses the ability to conserve the information about changes that took place long ago. For example, organisms that died several million years ago and turned out to be under definite specific conditions may conserve their shape. For example, in such processes as the formation of amber, carbonization, or zoolith formation, the composition of the organism changes completely but its shape is conserved and remains cognate. Due to this feature, it was established where ancient oceans and continents were situated on the Earth, how the continents drifted, when life appeared on the Earth, how living organisms evolved etc.

Material bodies may interact with other mechanically or through another method while they move. The results of these interactions may be diverse: scratches, deformations, magnetic or electrophysical changes on the surface of solids, the formation of new chemical compounds, acoustic or electromagnetic waves propagating in different directions etc. These are primary material data. These material data may be recorded with specially developed sensors or with the receptors of living beings. These recorded data are usually called virtual data. These data are not material any more; they are ideal. The virtual data may be subjected to changes and transformations in a definite space that is called the virtual space. Modern notion of information includes both primary and virtual data. The primary material data are quite clear because they are real; however, virtual ones are latent; their motion and transformations may be followed only on the basis of indirect data, for example on the basis of the results of action of sensors or living organisms. So, the virtual space may be defined as a state of a definite vector system formed in the analysis of the primary material data and allowing one to reach optimal decisions.

Living creatures may not only read the primary material data but they also may create these data themselves. A human being may write any virtual information on paper with the help of letters. If we consider only the material aspect of such writing, this will be only a sheet of paper spotted with paint in definite sites. o detect the primary data (letters) on such a sheet of paper, a person able to read is necessary. In the general case, for one man to pass any virtual information to another man, he should code it bring definite changes in the surrounding material world thus creating the primary experimental data. After that, another person who is able to read, understanding the language or gestures, should read these data, decode them, then form virtual information for himself on this basis. There is no other reliable route to pass the information from one person to another. We consider telepathy to be not proved.

For successful work with the information, it should be stored somewhere. Under normal conditions of our life, only solids may conserve the primary data. Gases and liquids do not possess this ability. The developed living organisms may conserve the information in the brain cortex neurons. However, it is still not very clear how this information is recorded and reproduced. The information is stored in computers in special hard disks. At the micro-level, where no notions of solid, liquid or gas exist, information may be stored due to the rigid structure of molecules. This possibility is used by living organisms; their genetic code is recorded in polynucleic acids with the help of four bases: adenine, guanine, uracile and cytosine. Considering smaller objects - atoms and atomic nuclei - we may only assume the possibility of using them for storage of primary data. These particles may exist in the excited states only for a short time, but some of these objects possess spin, a magnetic moment that can be conserved for rather long time. In order to use these effects, it would be necessary to develop an analogue of a writing device surely transferring the state of a separate atom from one to another, and an analogue of a reading device. In addition, one cannot exclude that on the global scale material objects may be similarly used to store the primary data. The galaxies are stable due to their internal rotation which is an analogue of spin in micro objects; it may be potentially used to store the information.

The major part of primary data about the surrounding world is obtained by us due to the ability of electromagnetic waves to propagate in vacuum, air and some bodies. If this were not the case, we would not get any idea of the Sun, starts, and the universe. However, we ourselves would not exist in this case because life exists on the Earth due to the energy of solar light. Energy is necessary to read, process and record data; so, while the moving matter possesses energy, it is possible to read the primary data, analyze them, compare, and form virtual spaces. So, motion of the matter at the same time creates the conditions for the appearance of virtual information spaces. Because of this, we may speak in our world of the motion of two worlds: the material one, and a virtual (ideal) one. The major difference between these worlds is the fact that the material world is scalable, that is, with an increase of material objects their mass changes; so does the intensity of their interaction and many other characteristics. Information is not scalable: analysis and processing of the information may be carried out with equal success with the help of computers in which the working elements differ in size. The larger are these elements, the larger place is occupied by the memory and the lower is performance speed. Because of this, objects with smaller elements are preferable for information storage and processing.

The primary material data recorded on some medium 
or transferred from one source to another can be characterized by total volume; its exact evaluation was developed by Shannon, C.E. However, total volume of a message does not indicate the amount of useful information contained in it. For example, a message may be composed of a random set of letters. Only qualitative criteria have been developed for evaluation of virtual data. Thus, several levels exist for characterization of these data: syntactic, semantic, logical, model description, and latent meaning. Only a message meeting definite rules of orthography will pass the syntactic control. The number of the kinds of syntactic control may be equal to the number of known languages including programming languages. But if a message has passed the syntactic control, this does not mean that it necessarily contains the virtual information of a higher level, because a message may be composed of unrelated sentences and thus having no semantic (meaning) content. Another kind of control is logical control; it establishes the absence of logical contradictions in the message. Then it may be determined whether a message is some model, or a law, or a general scientific discipline. Finally, a message can contain latent meaning. The latent meaning may be established at any level. For example, it a message does not pass the syntactic control with the help of a definite language, this does not mean that it does not contain useful information. It is quite possible that it contains the latent information; it may be detected through the syntactic analysis with the help of another language. The most vivid example containing latent information is the quatrains of Michel de Nostredame in which latent predictions were found and will be searched for.

Since information may be characterized quantitatively (primary data) and by several qualitative levels (virtual data), the same levels may be used to characterize also the spaces in which information processing is performed. For example, sensors that switch off the street lighting in morning respond to the amount of sunlight recorded by photoelectric cells. A primitive space with the quantitative evaluation of information is formed in these sensors. Spaces with either quantitative or qualitative information processing may be formed in computers. Spaces with different levels of information processing may be formed in living organisms. Humans possess even higher possibilities in this respect. They may not only process information but transfer it to each other and to forthcoming generations.

Let us consider the features of interconnection between the matter, a human being and human civilization as a union able to generalize and store virtual notions. During the whole history of mankind, civilizations were accumulators of primary data and generators of scientific notions. With a loss of some civilizations, the entire in- formation accumulated by them was lost. This information included the practical experience in agriculture and crafts, ceremonies and life conditions of different estates of the society, literature and scientific achievements, philosophy and religious doctrines. Today we may establish some facts concerning the vanished civilizations on the basis of conserved written sources and material values found during archeological digs. Even in this case the notions about those civilizations may be recovered only by people through consecutive transformations of the primary archeological data into virtual information and then, after its generalization, through the formation of our own notions about the life of people, their culture and their scientific doctrines. With the general approach, the motion of virtual information is formed specifically in the brain of each person. This motion may coincide with that of another person or differ from it. Because of this, the number of subjects possessing brain is more than six milliard (billion) while there is only one material world.

In general, the most surprising feature of the material world is the fact that the motion of the matter may promote the formation and long-term functioning of the structures able to read and analyze the primary data, then create virtual data, model representations and doctrines on this basis. At present, our civilization has achieved definite success in information processing and in the creation of scientific notions that are the basis for subsequent development of the possibilities of Mankind in the investigation of the surrounding world, in assimilation of natural resources and the improvement of the life level for each human being. Under definite conditions, Mankind is ready to share the entire set of data available and to carry out information processing for other civilizations; however, no other civilizations have been discovered so far. Below we will propose a model of the universe according to which Mankind will get the possibility in the future to process the information for an external supergiant civilization that had presumably created our world.

\section{COMPUTER-LIKE MODEL OF THE UNIVERSE AND THE POSSIBILITIES OF MANKIND}

We are going to propose the model of the Universe in which the main idea is a humanoid civilization able to process information and to generate doctrines about the nature. The basic provision will be formulated as follows: the Universe has been created in order that somewhere in it an intelligent civilization would appear that would be able to process the information. Because of this, the proposed model differs from the majority of the models of the Universe in which the role of unnecessary interior 
observer or at least a primer for a new Big Bang is assigned to Mankind. Our opinion may be expressed by paraphrasing the known words of $\mathrm{R}$. Descartes: $<<$ If we think, this excellent world has been created in order that we could think $>>$. For what reasons do we hold to this statement? We simply respect (appreciate) ourselves. We are surprised at astrophysicists who assign as miserable a role as a chancy observer to humans and therefore to themselves, too, in their models of the development of the Universe.

As we declare that the Universe has been created, then, there should be the creators of our world. We cannot define exactly what or who are these creators. Our notion of the creators is close to the ancient Greek polytheism or belief in twelve gods. But if there were creators, they had definite goals, and we are to understand these goals and help the creators to solve their problems. In order to understand what the creators assumed and hoped to obtain from us, it is necessary to consider what we can do in this world.

On the scale of our galaxy we can do nothing, simply because we will never get even to the centre of our galaxy. We make such a conclusion because the scientists have not invented a spaceship able to move faster than the velocity of light, a time machine or the possibility to travel outside our four-dimensional space-time. We also do not believe in the possibility to travel with the help of "wormholes" assumed by some astrophysicists [2,8]. We may only observe the Universe being interior indifferent observes but tending to have a definite probability in future to become able to predict the further development of the Universe. Mankind has achieved definite success in nature development of eth Earth. As far as the interstellar space is concerned, we stick to extremely pessimistic opinion.

Now we will consider the Solar system. Can we change the Solar system so that more people would be able to live in it? Assume that it is possible to move Mars and Venus by some miracle to the Earth's orbit. If the Earth, Mars and Venus move equidistant along one orbit, such a "necklace" of planets will be in quasi-equilibrium which may be sustained for arbitrarily long time. After some time, the climatic conditions on Mars and Venus travelling around the Sun along the Earth's orbit will approach the climatic conditions of the Earth. Then there will be three planets suitable for habitation. However, these changes in the Solar system are impossible. Then, what will Mankind be able to do in the Solar system? People will be able to travel by spaceships to Mars. Perhaps a settlement may be built there. Other planets of the Solar system will be studied most likely with the help of automatic stations. Mankind can observe the near-Earth space, calculate the motion of planets, as well as aster- oids and comets. Maybe this will allow us to protect he Earth from asteroids. However, Mankind is unable to perform any noticeable changes in the Solar system.

What changes are to be made on the Earth in order to provide the possibility to inhabit it with more people? The most favorable living conditions are those existing at the equator and in temperate latitudes. The conditions existing at the poles are unfavorable. Subtropical belts often contain deserts. The conditions in the mountains are also unfavorable for life. So, it is desirable that the polar and subtropical belts were covered with oceans, that no marshland regions or mountains were occurring on land. The size of main lands should not exceed 1000 $\mathrm{km}$, which would allow winds to deliver precipitation easily. However, at present Mankind is unable to reshape land and move continents. This can hardly be possible also in the future.

What can humans do at present? They can build cities and roads, disforest, plough up the steppe. Burning fuel, we enhance the greenhouse effect in the atmosphere, which may cause warming on the Earth. Operating nuclear power stations also cause warming. However, minor warming recorded at present on the Earth is most likely connected with an increase in solar activity. We are able to annihilate the majority of large animals and human beings using poisonous substances. People can change the reflection power of the atmosphere with the help of nuclear explosions, which would lead to Nuclear Winter and maybe to the destruction of human civilization. Nevertheless, we may hope that no catastrophes of this kind would ever happen on the Earth. Maybe this is a complete list of what we can do on the Earth. Yet this is still nothing on the universal scale. In other words, homo sapiens can do almost nothing in the physical world on the universal scale and therefore means nothing.

However, Mankind can not only make some changes to the material world but also create intellectual values. Now we will consider not so much the aesthetic capacity of homo sapiens as the possibilities of Mankind in information accumulating and processing. Mankind undoubtedly has achievements in this area that open unlimited possibilities. A great number of various doctrines has been created by people by present; people can carry out rather complicated calculations that allow predicting the behavior of matter, from the quantum size to the sizes of galaxies. Large amounts of information may now be stored, systematized and processed with the help of computers. In addition, the speed of computers and their memory are permanently increasing. We have not any principal obstacles that would limit the progress in computer systems. If we take into account the time that we have at our disposal while the Sun sustains the conditions favorable for life on the Earth, we will see that 
these possibilities are almost unlimited. By present, Mankind has created the global computer network, the Internet. Maybe Creators will be able to communicate with us through the Internet to pose some problems for solving that would be important for them. Surely, Mankind would undertake that work simply because of the gratitude for the creation of this surprising and tremendous world.

If our Universe had been created for sensible beings capable of data processing to appear in it, then, in turn, we also will be able to create the conditions that will allow self-formation of "sentient structures" at the micro-level, so that they would be able to process information but this time for us. In our opinion, this is the future of computer systems. Let us at first consider the stages of computer production allowing Mankind to create increasingly perfect computer techniques.

\section{TECHNOLOGICAL STAGES OF COMPUTER PRODUCTION}

The most important parameters characterizing any computer are its speed and memory capacity for storing executable programs, initial and intermediate data, and computation results. All these parameters are directly connected with the size of working elements. The smaller are these elements, the higher is computer speed. Several stages of computer production with their attempts to miniaturize the working elements may be distinguished.

The first stage of computer production is made by hands. At this stage, all the elements of the first computers, including electronic tubes or the first transistors, were manufactured manually. Assembling and soldering were manual, too. This stage, similarly to all the subsequent ones, had its own size limits. This limit may be illustrated by a horseshoe that a fabulous lefthander Levsha had manufactured to horseshoe a flea. This makes about $10^{-5} \mathrm{~m}$. Human hands are a too coarse instrument to make smaller details.

The second stage is due to solid crystals. The ability of solids to form the zones with definite easily adjustable electro physical characteristics and conserve them for a long time was employed at this stage. This allowed us to manufacture micro circuitry, memory elements with high pacing density. Of course, in these cases, too, everything is made by human hands, including the devices for manufacturing the circuit boards and computer assemblage, but the most delicate work is the formation of small zones of crystals with controllable size and required characteristics; this may not be done directly with hands.

The formation of micrometer- and sub micrometersized elements of integrated circuits (IC) is performed by means of lithography. Lithography is a method to form the required relief (pattern) during IC manufacturing process. The patterns are made either with the help of preliminarily manufactured templates (photographic methods) or by means of scanning electron (ion) beam controlled by computer. At present, depending on the required miniaturizing degree and expenses, photolithography in the visible spectral region is used (the achieved limit of element miniaturization is $1.0-2.0 * 10^{-6} \mathrm{~m}$ ), as well as UV lithography $\left(0.5-0.8 * 10^{-6} \mathrm{~m}\right)$, X-ray lithography $\left(0.1-0.5^{*} 10^{-6} \mathrm{~m}\right)$, electron beam lithography $(0.2-$ $\left.0.3^{*} 10^{-6} \mathrm{~m}\right)$, ion lithography with the beams of $\mathrm{H}^{+}, \mathrm{He}^{+}$, $\mathrm{O}^{+}, \mathrm{Ar}^{+}$ions $\left(0.1-0.5^{*} 10^{-6} \mathrm{~m}\right)$.

It has been established by present that further decrease in the size of solid crystal elements causes a decrease in the time within which the useful characteristics are conserved. The performance characteristics of modern integrated circuits may be conserved during many years, while the structures with smaller elements may retain the working state within a limited time (hours, minutes) due to dark currents, temperature fluctuations, microbreakdown, tunneling etc. To make the work of devices with these features possible, it is necessary to perform periodical updates of the parameters of working elements. This periodical interruption of computation process will take short time but total speed of these computers will be much higher than that of the presently existing computers due to higher miniaturization. This problem seems to be one of the most important reasons that would restrict further miniaturization of solid crystal computers. The miniaturization limit achieved by means of beam lithography is estimated as $10^{-7}-10^{-8} \mathrm{~m}$. It should be noted that almost all the possibilities of beam lithography have already been involved by present.

Further progress in miniaturization is considered to be connected with the possibilities of scanning tunneling microscope (STM). Surface modification may be performed by means of STM either through the direct mechanical action of a needle on the surface (direct scratching) or by local electrochemical oxidation of the surface with the help of a needle. Separate deformed or oxidized regions with a size of $1-2 * 10^{-8} \mathrm{~m}$ have been successfully obtained by means of STM [11]. However, these are only early attempts to apply this method to modify crystal surface.

The third stage is molecular. Definite success at this stage belongs only to living organisms at present. However, Mankind in theoretical and technological development has already come right up to the possibility of building a molecular computer. A large number of small molecules are known to be capable of reversible rearrangements under the action of radiation or magnetic field. These rearrangements are either geometric changes of a molecule or magnetic and/or charge redistribution. All these effects may be used in instantiation processors 
or memory elements [12]. Yet, it should be stressed that the problems connected with the conservation of performance characteristics of elements will become even more acute in molecular computers. The limit of miniaturization of molecular computers should be determined by the size of molecules being potentially able to rearrange reversibly. An example of such a size may be the size of the aromatic ring. The distance between the opposite atoms in benzene molecule is $2.9 * 10^{-10} \mathrm{~m}$; taking into account the Van der Waals radii of atoms, the radius of benzene molecule is equal to $\sim 1^{*} 10^{-9} \mathrm{~m}$. The latter size may be accepted as the limit of miniaturization of a molecular computer.

The fourth stage is assumed to be atomic. Assumptions concerning quantum computers are also put forward; these computers are to employ different states of electron spins [13]. However there are no real models of computers applying on interatomic effects. It should be noted that the size of atoms is $10^{-11}-10^{-10} \mathrm{~m}$.

The common phenomenon for the four computer schemes considered above is energy flux through the elements of the system. In modern computers, it is electric current. A computer in which the driving force is a light wave or liquid may also be possible. All these design may be called static models of computers with rigid structure. Self-assemblage and self-formation are increasingly frequently used in descriptions of manufacture of IC elements; thus it is stressed that automatic machines create only definite conditions in micro-zones, while the useful electro physical properties are formed in them due to the special properties of solid bodies or crystals. Will it be possible in our material world to create conditions for self-formation of automatic machines themselves, that would be able to manufacture superminiature computers? We think this is possible. To confirm this statement, let us consider our modern civilization on the Earth. Potentially, Mankind is already able to process information for extraterrestrial consumers. That is, our planet is an analogue of a computer. If the size of the Earth is considered as the size of a computer, the elements on which data processing is carried out are super miniature. Some conditions are necessary for the self-formation of such a computer; these conditions have occasionally formed in the Solar system: the source of energy is the Sun, the material substance on which life had begun is the Earth's surface, energy absorber is the universal space. This model, unlike the static one, will be called the dynamic computer model. While the static computer model is a device in which data processing is performed on specially built easily adjustable elements, the dynamic computer model is a black box which is permanently improving under the action of long energy action and finally becomes able to perform data proc- essing. The majority of material objects that are subject to energy action simply scatter the energy without forming any structures able to process the data. However, we think that in some cases it will be possible to develop a device working according to the dynamic scheme.

In our opinion, similarly to the developing civilization, self-structuring substance in the dynamic computer scheme will always get adjusted to the problem to be solved, and it will itself try to find more efficient and fast solution methods. This means that it is not necessary to develop special computer languages and to write various data processing programs. Other problems arise; they are connected with the probability of formation of a selfstructuring substance, maintenance of its active capacity for work, and the possibility of information connection with it.

\section{POSSIBLE SCHEMS OF DYNAMIC COMPUTERS}

The major physical conditions are: 1 - artificial maintenance of the unstable state of the material substance, 2 - the possibility of additional action on this substance, and 3 - the possibility to receive responses to these actions. The simplest example of the dynamic computer model may be laser generator. Another example of the possible dynamic computer model may be the scheme of the Electron-Positron Collider (EPC). Recently constructed Large Hadron Collider (LHC) potentially may also serve as a model of the dynamic computer. It is quite possible that the Big Bang was initiated by a collision of two gigantic parts of the substance [2] produced according to the EPC or LHC schemes. In addition, other schemes of dynamic computers are quite possible, too. Even now we can try to make experiments in this direction. It may turn out that the development of a dynamic computer is much simpler and cheaper than, say, a molecular computer. It should be noted that the development of a dynamic computer is to a definite extent equivalent to the creation of sentient life in the Universe. During the whole history of Mankind nothing similar has even been created by humans. However, it should be noted that the creation of sentient life is not quite novel idea. It has been under discussion in science fiction since long ago.

The largest problem of any dynamic computer is the possibility of rapid information exchange between the active working zone and the external customers. Information may be reliably transferred in our world only in the form of primary material data; their maximal rate is limited to the velocity of light, which is too slow on the universal scale. These are the major shortcomings of the model proposed by us. But why did the Creators make our world in which the information cannot be transferred 
infinitely quickly? The matter is not so simple, however. Restricted maximal rate of the transference of material objects has substantially increased the lifetime of the Universe. If the velocity of matter expansion could be infinitely high, there would possibly be lack of time for the generation and development of life on the Earth.

There still remains a hope that the tools of information transfer with the velocities higher than the velocity of light may be found. An interesting communication that appeared in 2008 was made by Nicolas Gisin and coworkers from the University of Geneva [14]. They demonstrated that the entangled photons spaced at a distance of $18 \mathrm{~km}<<$ sense $>>$ changes in the states of each other. The rate of correlation of their behavior exceeds the velocity of light by several orders of magnitude. The authors proposed to call this effect $<<$ the transfer of quantum information $>>$. It is still not clear yet whether it is possible to use this effect for transferring any data. We have mentioned previously that we do not believe in the possibility to move material objects with the help of the assumed wormholes [8], but maybe it will be possible to transfer the information.

So, we may be useful for the assumed Creators due to the increasing possibilities of Mankind in data processing and due to the large experience in modeling and generating scientific concepts. Along with the possibilities, Mankind has also got several problems. The major one is connected with the lack of energy for further accelerated development. This problem will only become more acute in the future. It is quite possible that the Creators will be able to supply Mankind with energy (which is so necessary for us) as a gratitude for data processing. Then there will be mutually beneficial collaboration between the assumed Creators and Mankind.

\section{ASSUMPTIONS CONCERNING THE CREATORS IN RELIGIOUS AND SCIENTIFIC DOCTRINES}

The idea of a Creator, or Creators of our world seems to be the most ancient, the most disputable one, and still unsolved till present days. We do not want to discuss this problem; we will only set out our opinion on the origin and development of this idea. The life of a human being in this world is very diverse. Sometimes the actions of people lead to a desirable result but sometimes they do not. In the cases when the activities of people had not brought them up to the desirable results for unknown reasons, prerequisites for assumptions concerning external powerful forces appeared. At the earliest stage of the development of human civilization, people were unable to explain many frightening natural phenomena, such as earthquakes, tsunami, hurricanes, thunderstorms with lightning, droughts and flood events, illness etc. There- fore, people interpreted the world as hostile and frightening. People needed support and a strong protector. It was that time when the religious doctrines appeared; in those doctrines, the Almighty Creators or God created the Earth in the centre of the world for people to be able to live on it. All the disasters and frightening natural phenomena were understood just as punishments for disobedience or bad actions. The Creators might be propitiated with prayers, good deeds, or sometimes with sacrifices.

Centuries had passed. Many natural phenomena were explained by natural reasons. Due to the accumulated knowledge, Mankind was able to develop the methods of protection from the negative natural phenomena. The notions about the external world had changed essentially, too. The Earth turned out to be not the centre of the world but just one of the planets of the Solar system; the Sun is situated not in the centre but at the very edge of our vast Galaxy. There are many other galaxies in the Universe. In addition, it had not been established where the Creators can be found in our world; no reliable information connection with them was established. At that time, religious notions were replaced by materialistic ideas completely denying the real existence of the Creators of the Universe. According to the classical materialistic ideas, Mankind is only an insignificant observer that has appeared by chance within a small time interval of the development of the Universe.

Some more time passed by, and the possibilities of Mankind changed substantially, especially in the area of data processing, systematizing and modeling. In addition, any restrictions that might be limiting the progress in this direction are not seen. At present, Mankind is able to process the information for any possible consumers. That is why we regenerate the idea of external Creators of our world who presumably could have the need for rapid data processing.

In the present work, we did not try to unite or conciliate the materialistic and idealistic directions in philosophy. Sticking to the idea of the primary character of the matter, we tried to give an answer to the question what our world had been created for, and what Mankind can do in this world. To answer these questions, we regenerate a well known idea of the Creators of our Universe at a novel level. Every reader may agree with such an approach or, quite contrary, consider it to be incorrect.

\section{CONCLUSIONS}

In most of the known hypotheses of the Peace development the mind in the Universe is an unnecessary attribute. In the proposed computer-like model namely mind and humanoid civilization is the main purpose of the Universe. This model is based on the assumption that 
the external Creators organized the Big Bang, which became the cause of our universe. The pragmatic goal of the Designers is the formation of a material substance that can process information. The proposed computerlike model of the Universe in the future involves communication with the creators to do their computing orders. However, this model of the world is not perfect due to lack of an infinitely high speed of information transmission. Maybe the with the opening of new data on the Nature or the development of technical capabilities of the Mankind it will became possible to propose the World model, fully consistent with the data about the world and the increasing possibilities of our civilization in the development of nature, and especially in the field of information processing.

\section{ACKNOWLEDGEMENTS}

The author thanks Prof. V. I. Belevantsev and Prof. I. K. Igumenov for discussion of this paper.

\section{REFERENCES}

[1] Cherepaschyk, A.M. and Chernin, A.D. (2009) Cosmology: Discoveries and questions. Nauka iz Pervyh ruk (Science First Hand), Novosibirsk, 1, 26-37.

[2] Turner, M. (2009) The origin of the universe. Scientific
American, 9, 17-23.

[3] Cherny, V. (2009) Cyclical Universe. Nauka $i$ Zizn (Science and Life Magazine), 10, 40-43.

[4] Bojowald, M. (2009) Big bang or big bounce? Scientific American, 1, 18-24.

[5] Djenkins, A. and Peres, G. (2010) Looking for life in the multiverse. Scientific American, 1, 15-23.

[6] Spiridonov, O.P. (1991) Fundamental physical constants. Vyschay Shkola (in Russia), 238.

[7] Karshenboim, S.G. (2008) New recommended values of the fundamental physical constants. (CODATA 2006) Physics-Uspekhi, 51, 1057-1064. doi:10.1070/PU2008v051n10ABEH006668

[8] Chirkov, J. (2008) Upon investigation secrets of universe. Nauka v Rossii (Science in Russia), 2, 61-62.

[9] Frolov I.T. (2001) Philosophical dictionary. Moscow.

[10] Islinsky A.J. (1980) Polytechnic dictionary. Moscow.

[11] Aseev, A.L. (2007) Nanotechnology in solid-state electronics. Novosibirsk.

[12] Rambidy, N. (2006) Nanotechnology and molecular devices. Nauka v Rossii (Science in Russia), 6, 38-46.

[13] Aaronson, S. (2008) The limits of quantum computers. Scientific American, 3, 62-72. doi:10.1038/scientificamerican0308-62

[14] Salart, D., Baas, A., Branciard, C., Gisin, N. and Zbinden, H. (2008) Testing the speed of spooky action at a distance. Nature, 454, 861-864. doi:10.1038/nature 07121 